ARCHIVO ESPAÑOL DE ARTE, LXXX, 318

ABRIL-JUNIO 2007, pp. 177-206

ISSN: 0004-0428

\title{
VARIA
}

\section{NUEVOS PAISAJES DE CARLOS DE HAES}

El objeto de esta breve reseña, es el de dar a conocer cuatro nuevas obras del gran maestro del paisaje realista español Carlos de Haes, que, por su calidad pictórica -dentro del conjunto de las obras de este pintor, de prolífica producción, que van apareciendo-, bien creo que merecen aunque sea un pequeño estudio.

Estos cuatro cuadros que traemos del célebre paisajista son óleos sobre lienzo, aparecidos recientemente en una colección particular alicantina, y de los que tenemos que especificar que son obras de composición, perfectamente acabadas, realizadas en su estudio por el artista a base de bocetos y apuntes tomados del natural; cuadros de buen formato, concebidos por el pintor expresamente para un mercado de rica clientela (ya fuese aristocrática o burguesa) que demandaba el tipo de paisaje por él puesto de moda en la España de la segunda mitad del siglo XIX, y que adquiría estos decorativos cuadros para adorno de sus residencias ${ }^{1}$. Se trata, pues, de paisajes panorámicos de buen tamaño, y no de bocetos o estudios de los muchos que realizó a lo largo de su vida y de los que dejó a su muerte gran cantidad a sus discípulos que, a su vez, los legaron al extinto Museo de Arte Moderno, legado con el que se creó una "sala Haes" de escasa duración, ya que pronto fueron dispersados (mediante depósitos de esa entidad), por museos provinciales y otros organismos oficiales de toda España. Situación ésta heredada por el Museo del Prado cuando, al extinguirse el Museo de Arte Moderno, pasaron sus colecciones del siglo XIX a dicho museo, viniendo a constituir así esta amplia colección de estudios y dibujos el escaparate más conocido (y, a veces, equívoco) de la obra de Haes. A este respecto, pues, hay que dejar claro que, una cosa son los bocetos y estudios instantáneos del artista, realizados frente al natural, generalmente de factura suelta, manchista, de gran frescura y espontaneidad, de magnífica captación atmosférica y luminosidad, y, por todo ello, cargados de modernidad; y otra cosa sus cuadros de empeño, primorosa y detalladamente elaborados, compuestos en su estudio, de corte más académico, a pesar de su realismo; cuadros a los que se refiere Díez en estos términos: "testimonios extraordinariamente elocuentes de las mejores cualidades de Haes

\footnotetext{
1 Díez, J. L., "Introducción”, en Ana Gutiérrez Márquez, Carlos de Haes en el Museo del Prado (1826-1898).Catálogo razonado. Museo Nacional del Prado, Madrid, 2002, p. 10.
} 


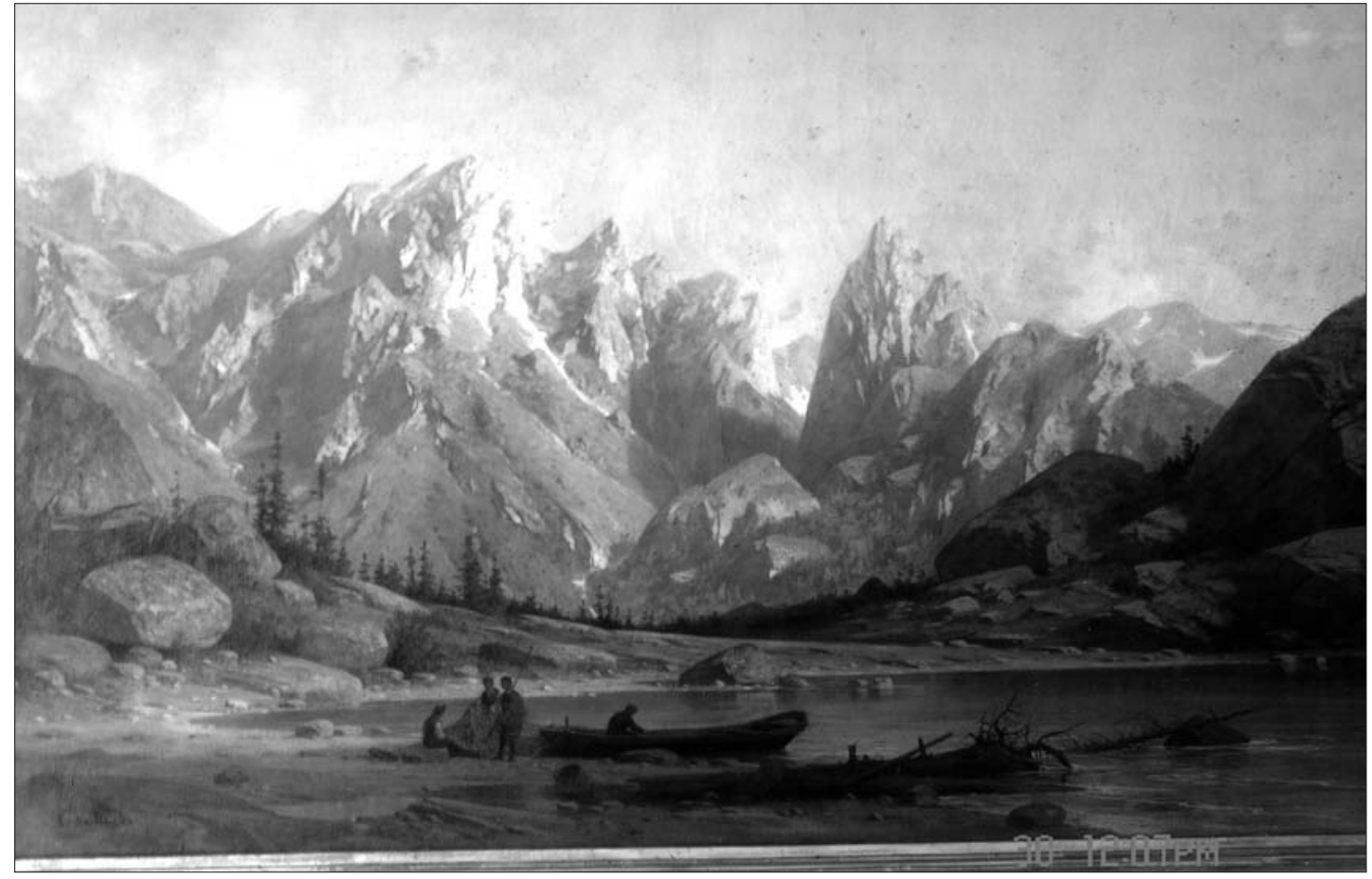

Fig. 1. Carlos Haes: Paisaje lacustre de alta montaña con pescadores. Colección particular.
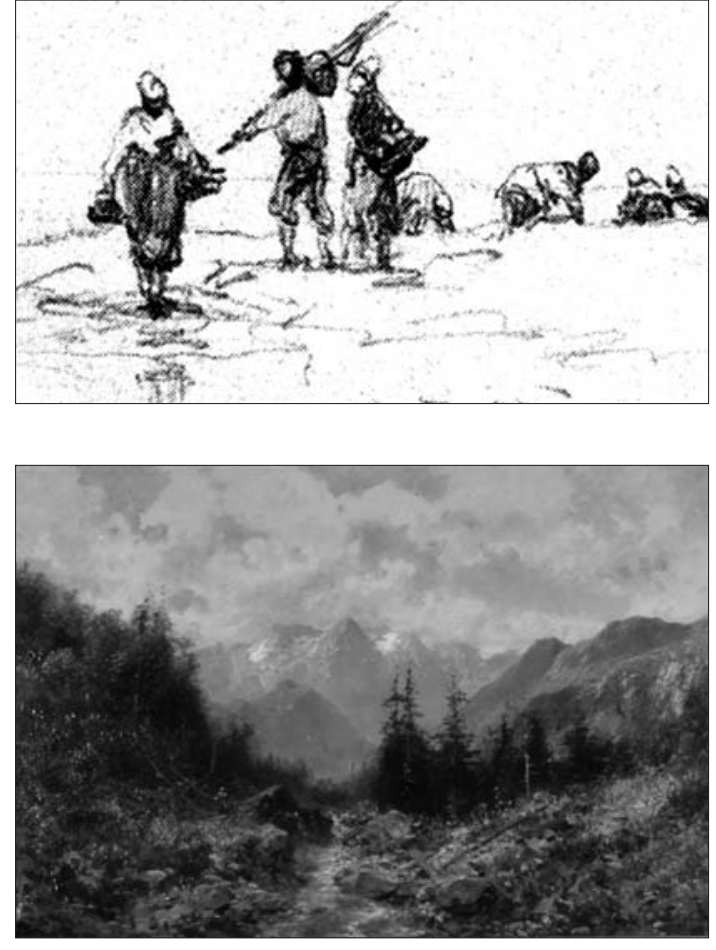

Fig. 2. Carlos Haes: Pescadores. Museo del Prado. Madrid.

Fig. 3. Carlos Haes: Los Picos de Europa (?). Colección Rudolf Gerstenmaier. 
como verdadero maestro del género, muy por encima del resto de los paisajistas españoles de su generación" 2 .

El primero de ellos, que titulamos descriptivamente Paisaje lacustre de alta montaña con pescadores (Fig. 1), es un óleo sobre lienzo de bastante buen tamaño, ya que mide 100 x $180 \mathrm{~cm}$., firmado en el ángulo inferior izquierdo "C. de Haes". Se trata, por tanto, de un gran lienzo de composición, que nos muestra una espectacular vista panorámica de un alto valle de montaña con laguna: al primer término la orilla con el tronco de un árbol derribado y cuatro pescadores con una barca; de ellos, tres están en tierra faenando con una red, uno de estos con una caña al hombro, mientras que el cuarto se encuentra sentado en el bote. Este grupo de tres pescadores en tierra es muy similar a los que aparecen en los dibujos del pintor titulados Pescadores (Fig.2) (D-5280, Museo del Prado. Madrid) y Pescadores. Apunte de barca (D-5303, Museo del Prado. Madrid) ${ }^{3}$. La vista está encuadrada por el artista con sentido armónico, equilibrio y serenidad ambiental, siendo elementos fundamentales para ello el mantener la consonancia de líneas verticales y horizontales en la estructuración de la obra, al estilo de los paisajes clásicos flamencos del siglo XVII; radiografía o esquema imprescindible para lograr ese perfecto sentido de equilibrio, esa armonía que reina en la composición. Equilibrio y armonía que tienen su complemento en esa serenidad ambiental determinada por el estudio atmosférico y lumínico que flota en el ambiente y que establece la suave gradación de las lejanías paulatinamente hacia el fondo del cuadro, ayudado todo ello por la magistral utilización de los juegos progresivos de luces y sombras con las matizaciones del colorido. Todo ello se complementa con una aguda observación de los elementos naturales que conforman el paisaje, que sabe traducir y transmitir con gran realismo, pero envueltos en una poética de exquisito lirismo.

No sabríamos decir de qué lugar se trate, ni tan siquiera de qué cordillera. Pudiera ser de los Picos de Europa, del Pirineo o, quizá, podría pensarse también (y no sería descabellado) que sea una composición de taller organizada con diversos apuntes o bocetos tomados del natural. En definitiva, que pudiera tratarse de una construcción poética de cierta fantasía organizada con elementos captados de la realidad, algo que, a pesar de su pretendido realismo, conserva todavía resabios románticos. Pues, aunque Haes anduvo por los Picos de Europa y el Pirineo entre 1872 y $1875^{4}$, el hecho de que se trate de una obra realizada en su estudio, y perfectamente acabada, indica que muy bien pudiera haber sido pintada por el artista en fechas muy posteriores a esas, utilizando para su composiciòn bocetos tomados por aquél entonces "in situ" y que él conservaría en su estudio. Lo único que nos puede dar una relativa pista de la época de su posible ejecución es que el estilo pictórico del cuadro coincide con el que Cid Priego ${ }^{5}$ estableció, basándose en Aureliano de Beruete $^{6}$, para la que él denominó su tercera , y última, etapa pictórica, en que Haes abandona la alegre brillantez de colorido que dominó en su segunda etapa, adoptando un color menos brillante, pero de mayor transparencia, mostrando más gusto por los tintes apagados, lejanos al anterior gusto por la luz meridional, pero adoptando un vigoroso efecto, un colorido jugoso y una factura excelente y suelta, características que son perfectamente aplicable a esta obra. Así, dentro de

\footnotetext{
${ }^{2}$ Ibid.

3 Gutiérrez Márquez, A., Carlos de Haes en el Museo del Prado (1826-1898).Catálogo razonado. Museo Nacional del Prado, Madrid, 2002, pp. 296-297 (nº D-5280), y pp. 298-299 (nº D-5303).

4 Cid Priego, C. Aportaciones para una monografía del pintor Carlos de Haes, Instituto de Estudios Ilerdenses, Lérida, 1956, p. 70; C. Pena, M. C., El paisaje español del XIX: del naturalismo al impresionismo, Universidad Complutense, Madrid, 1982, pp. 195-198; Arias Angles, E., "Sobre tres paisajes de alta montaña de Haes", Archivo Español de Arte, t. LXXV, no 297, pp. 57-58.

5 Cid Priego, C., Aportaciones para una monografía del pintor Carlos de Haes, Instituto de Estudios Ilerdenses, Lérida, 1956, pp. 18 a 22 y 69 a 71.

6 Beruete, A. de, "Carlos de Haes", La Ilustración Española y Americana, Madrid, 30 de junio de 1898, año XLII, núm. XXIV, pp. 379 a 383.
} 


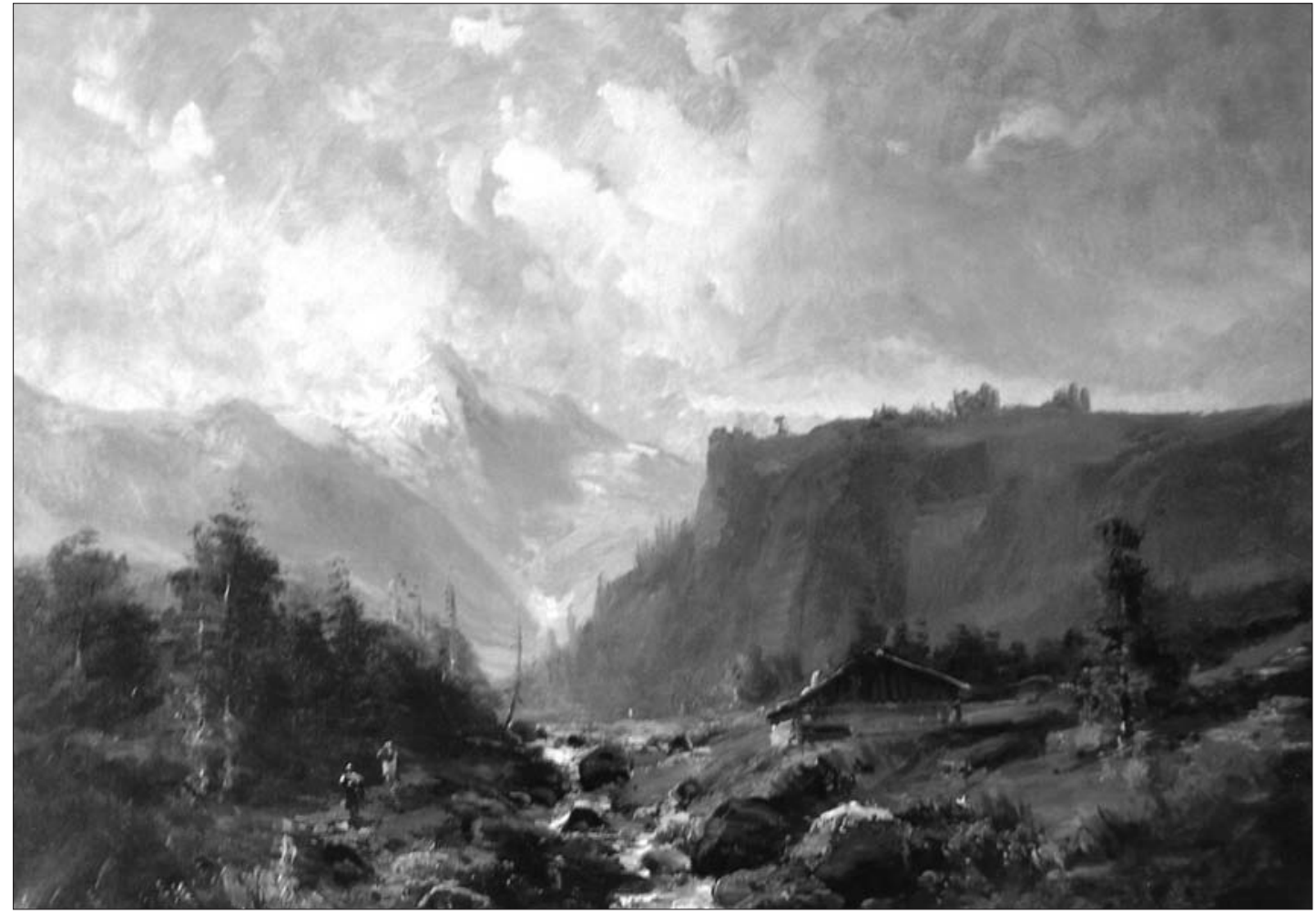

Fig. 4. Carlos Haes: Paisaje montañoso con torrente, cabaña y personajes. Colección particular.

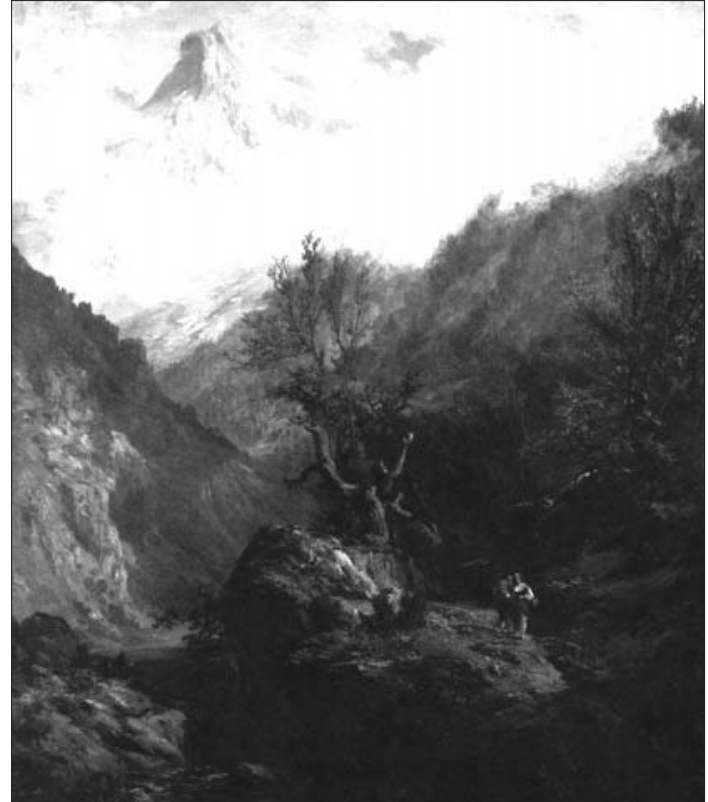

Fig. 5. Carlos Haes: Montañas de Asturias. Museo de Arte Moderno. Barcelona.

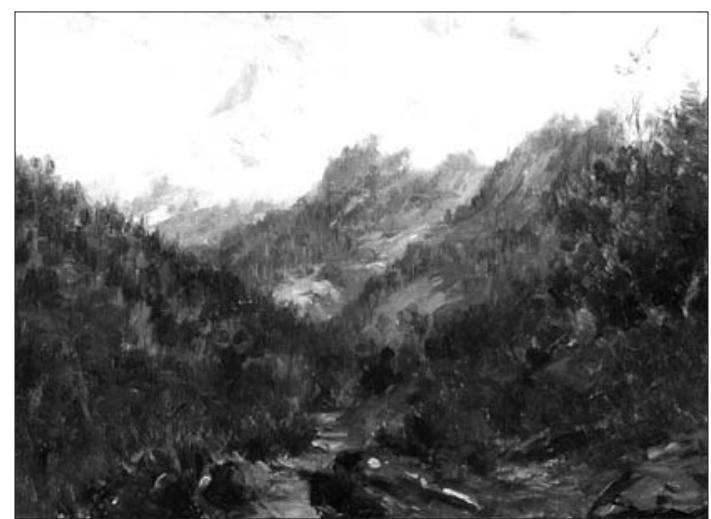

Fig. 6. Carlos Haes: Pirineos franceses. Museo Jaime Morera. Lérida. Depósito del Museo del Prado. 
esta amplia e imprecisa última etapa de su producción pictórica, fechable aproximadamente entre 1878 y 1890, creemos nosotros que puede ser básicamente datado el cuadro.

Por otro lado, son claras las similitudes en la técnica con que están realizados los pinos de alta montaña que aparecen en los términos medios de este cuadro, con los que igualmente vemos en otros cuadros de Haes ya publicados por nosotros, como en los que titulamos Vista de cordillera y Los Picos de Europa (?) (Fig.3), ambos de la colección Rudolf Gerstenmaier, o Paisaje de alta montaña con río y pastora y Paisaje montañoso con río y cabaña, los dos en el comercio ${ }^{7}$. De éstos, los tres primeros los clasificamos, en su día, en la segunda etapa del artista, y el cuarto en la tercera, según la división que de su obra hicieron Beruete y Cid Priego ${ }^{8}$. Son pinos de factura muy característica, algo desdibujados y borrosos en su media distancia y, quizá por ello, el artista opta por esa imprecisión en la composición de sus ramas, a base de manchitas sueltas, desvaídas, que las hace resultar algo etéreas, flotantes, en su estructuración.

El segundo de estos cuadros que presentamos de Haes, es también un óleo sobre lienzo de cierto tamaño, 89 x $116 \mathrm{~cm}$., firmado "C. de Haes" en el ángulo inferior izquierdo, y que titulamos Paisaje montañoso con torrente, cabaña y personajes (Fig. 4). Nos representa otro valle de alta montaña, dividido en dos por un pequeño torrente o arroyo que viene del fondo y desemboca en el primer plano al centro del cuadro, viéndose en su parte derecha una casuca junto a la torrentera, y en su lado izquierdo dos personajes que deambulan entre la corriente de agua y un bosquecillo; al fondo, altas montañas, quedando centrada la torrentera con el más alto picacho de las mismas, marcándose así el eje vertical de composición de la obra, estructura ésta que, con variantes, se repite en algunas obras de Haes. En efecto este tipo de composición recuerda mucho a la del cuadro Montañas de Asturias (Fig. 5), del Museo de Arte Moderno de Barcelo$\mathrm{na}^{9}$, si bien el formato vertical de éste hace que se reduzca mucho la panorámica horizontal con respecto al que tratamos, pero, en base, los dos poseen similar estructura, añadiéndose, además, la afinidad en los celajes de ambos en lo concerniente a la factura de las nubes. Esta semejanza estructural se acentúa aún más si comparamos el boceto general, que sirvió de base compositiva al cuadro del museo de Barcelona, con el cuadro que estamos estudiando, y que con el título de Pirineos franceses (Fig. 6) se conserva como depósito del Museo del Prado en el Jaime Morera de Lérida ${ }^{10}$. No hay duda de que, aunque no se trate del mismo lugar, el esquema básico compositivo es el mismo con las correspondientes y lógicas variantes, como podemos apreciar en las fotografías.

En cuanto a la posible datación de la obra, tenemos que recurrir nuevamente a la evolución de su técnica pictórica, según la secuencia de tres etapas o períodos en que dividieron su obra Beruete y Cid Priego ${ }^{11}$, porque la temática del asunto nada puede aportarnos, debido a que se trata de un cuadro de composición realizado en su estudio por el artista, generalmente a base de bocetos o apuntes tomados del natural a lo largo de su vida y que podía emplear en cualquier momento para la realización de un tipo de obra académicamente muy acabada. Por tanto, el vivo y alegre

7 ArIas Anglés, E., "Sobre tres paisajes de alta montaña de Haes", Archivo Español de Arte, t. LXXV, núm. 297,p. 60 (fig.3); Arias Anglés, E., "Nuevas obras de Carlos de Haes y Aureliano de Beruete", Archivo Español de Arte, t. LXXVIII, núm. 311, pp. 298 (figs. 1 y 2) y 301 (Figs. 3 y 4).

8 Vid notas 5 y 6.

${ }^{9}$ Catàleg de pintura segles XIX i XX. Fons del Museu d'Art Modern, Ajuntament de Barcelona, Barcelona, 1987, t. I, p. 484, n 1116; ARIAS ANGLÉs, E.(comisario), Tres grandes maestros del paisaje decimonónico español. Jenaro Pérez. Villaamil, Carlos de Haes, Aureliano de Beruete, Centro Cultural del Conde Duque, Madrid, 1990, pp. 266-267.

10 Arias Anglés, E.(comisario), Tres grandes maestros del paisaje decimonónico español. Jenaro Pérez Villaamil, Carlos de Haes, Aureliano de Beruete, Centro Cultural del Conde Duque, Madrid, 1990, pp. 332y 333; GutiérREz MÁrQueZ, A., Carlos de Haes en el Museo del Prado (1826-1898).Catálogo razonado. Museo Nacional del Prado, Madrid, 2002 , pp. 236-237 ( $\left.\mathrm{n}^{\circ} 7485\right)$.

11 Vid notas 5 y 6. 


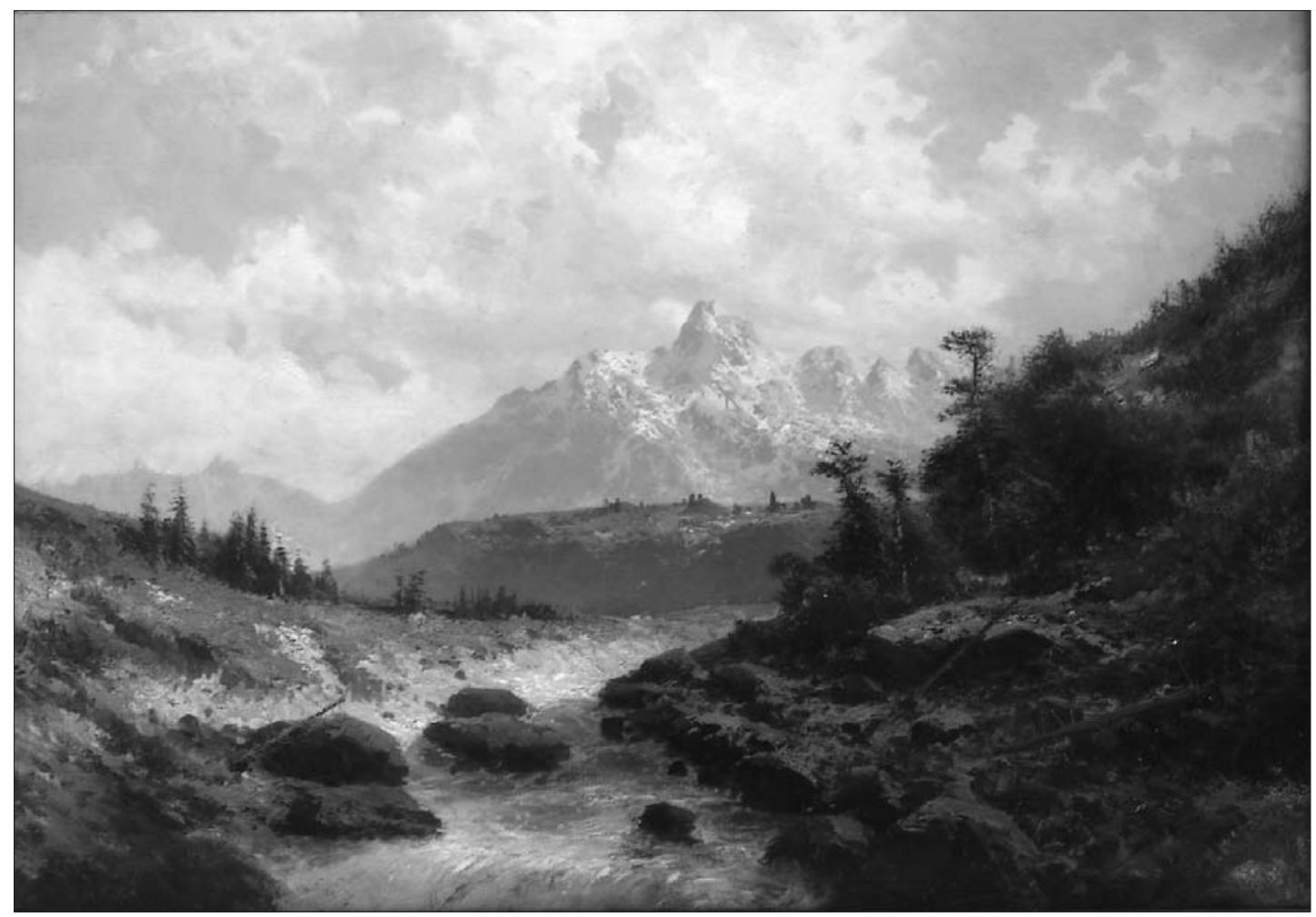

Fig. 7. Carlos Haes: Paisaje montañoso con torrente. Colección particular.

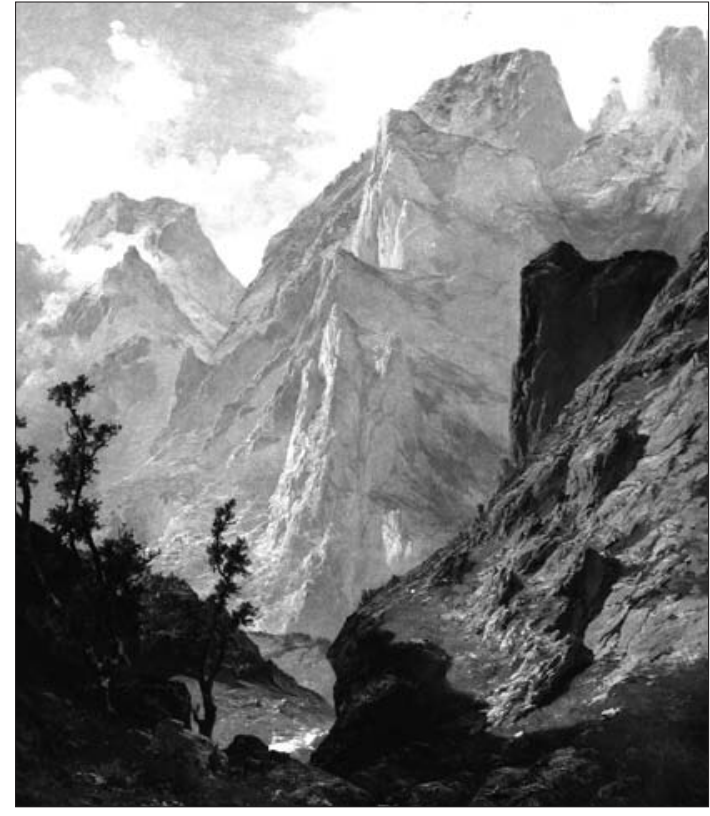

Fig. 8. Carlos Haes: Canal de Mancorbo en los Picos de Europa. Museo del Prado. Madrid.

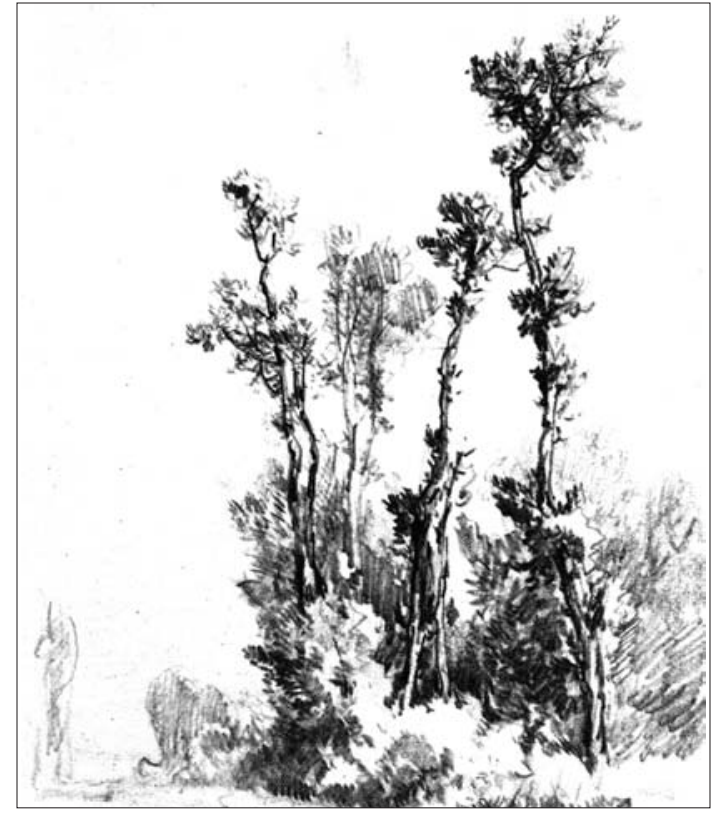

Fig. 9. Carlos Haes: Varengeville. Museo del Prado. Madrid. 
colorido de este cuadro, junto a lo suelto de su factura, nos hacen pensar que se trate de una obra de la llamada por Beruete y Cid Priego ${ }^{12}$ su segunda etapa pictórica, fechable entre 1870 y 1880 , en la que utiliza un colorido más jugoso y una factura más suelta que en su primera etapa.

Por lo que respecta a los dos últimos cuadros de Haes que traemos, hemos de precisar que forman pareja, teniendo las mismas dimensiones y procediendo también de la misma colección. El primero de ellos es un óleo sobre lienzo, de 65 x $92 \mathrm{~cm}$., firmado "C. de Haes" en el ángulo inferior izquierdo, y que citaremos primero por tratarse de un paisaje de montaña de similar estructura que el anterior que hemos visto. Lo titularemos Paisaje montañoso con torrente (Fig. 7), pues, en efecto, nos representa también un paisaje montañoso dividido en dos por un pequeño torrente o arroyo que, viniendo del fondo, desemboca en el primer plano al centro del cuadro, formando así un eje con el alto picacho montañoso que domina el horizonte de la composición. A un lado y al otro del mismo, como pantallas referenciales de marcar la distancia, con juegos de luces y sombras incluidos, dos pendientes arboladas; se marca aún más la referencia espacial hacia los fondos mediante otra elevación central que, a media distancia de la composición, y jugando también con las luces y las sombras, nos conduce la vista hacia el interior del cuadro, hacia su horizonte de alta montaña.

Aquí, en esta obra nos encontramos otra vez, en la ladera de la izquierda del riachuelo, con el tipo de pinos de alta montaña a que hicimos referencia en la primera obra que hemos presentado, y que titulamos Paisaje lacustre de alta montaña con pescadores (Fig. 1), siendo claras las similitudes en la técnica con que están realizados los pinos de alta montaña que aparecen en los términos medios de ambos cuadros, y que coinciden también con los que igualmente vemos en otros cuadros de Haes ya publicados por nosotros, a que antes nos referimos [Vista de cordillera y Los Picos de Europa (?) (Fig. 3), ambos de la colección Rudolf Gerstenmaier, o Paisaje de alta montaña con río y pastora y Paisaje montañoso con río y cabaña, los dos en el comercio $\left.{ }^{13}\right]$. Son pinos, como dijimos, de factura muy peculiar, algo desdibujados y borrosos en su media distancia, caracterizados por esa imprecisión en la composición de sus ramas, a base de manchitas sueltas, desvaídas, que las hace resultar algo etéreas, como flotantes, inconfundibles. Pero, en la ladera de la derecha del torrente nos encontramos con otro tipo de árboles, patentes también en algunos cuadros de Haes; se trata de arboles para nosotros difíciles de precisar, de troncos no muy gruesos, algo retorcidos, pero que poseen, a pesar de ello, cierta esbeltez y, sobre todo, una frondosidad algo escasa que hace visible, entre la parca hojarasca, la estructura de sus troncos y escasas ramas, rematados por copas de forma imprecisa, como penachos algo solitarios e indeterminados. Basta comparar estos árboles del cuadro que estudiamos, con los que aparecen en la parte baja izquierda del célebre cuadro del pintor Canal de Mancorbo en los Picos de Europa (Museo del Prado. Madrid) (Fig. 8), para darnos cuenta de ello. Se trata, sin duda, de las mismas estructuras vegetales, de equivalente forma y realizadas con análoga técnica. Similares son también a los árboles de troncos retorcidos que aparecen en el dibujo del pintor titulado Villerville (D-5301, Museo del Prado. Madrid) ${ }^{14}$; y, sobre todo, guardan una mayor similitud, con los árboles del cuadro que estudiamos, los del mismo tipo que aparecen en otro dibujo del artista titulado Varengeville (Fig. 9) (D-5252, Museo del Prado. Madrid) ${ }^{15}$, casi de idéntica estructura, podríamos decir, a los de este cuadro. Además de esto, queremos también precisar la similitud que existe entre la montaña que aparece en el fondo de este cuadro con la que pinta el artista en el estudio de hacia 1874 que se titula Montañas del Puerto de Pajares (Fig.10) (depósito del Museo del Prado en el Pazo-Museo Municipal de

\footnotetext{
12 Ibid.

13 Vid nota 7.

14 Gutiérrez Márquez, A., Carlos de Haes en el Museo del Prado (1826-1898).Catálogo razonado. Museo Nacional del Prado, Madrid, 2002, pp.266-267 ( ${ }^{\circ}$ D-5301).

15 Ibid., pp.308-309 (nº D-5252)
} 


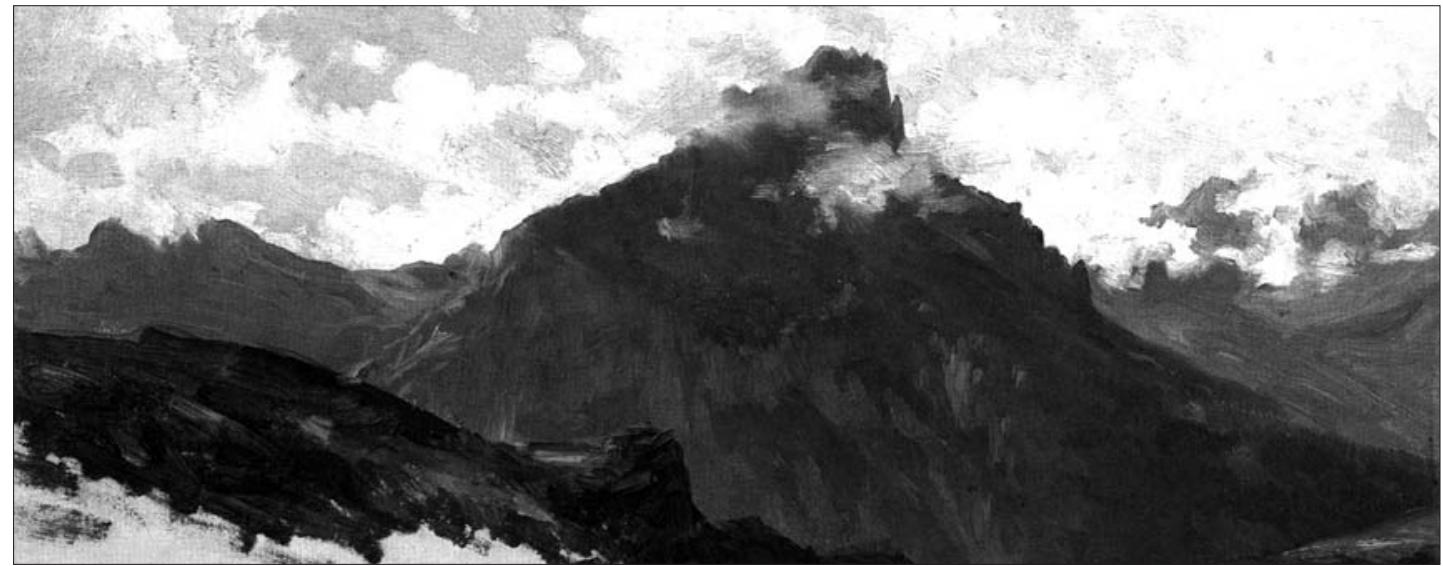

Fig. 10. Carlos Haes: Montañas del Puerto de Pajares. Pazo-Museo Municipal de Quiñones de León, Vigo (Pontevedra). Depósito del Museo del Prado.

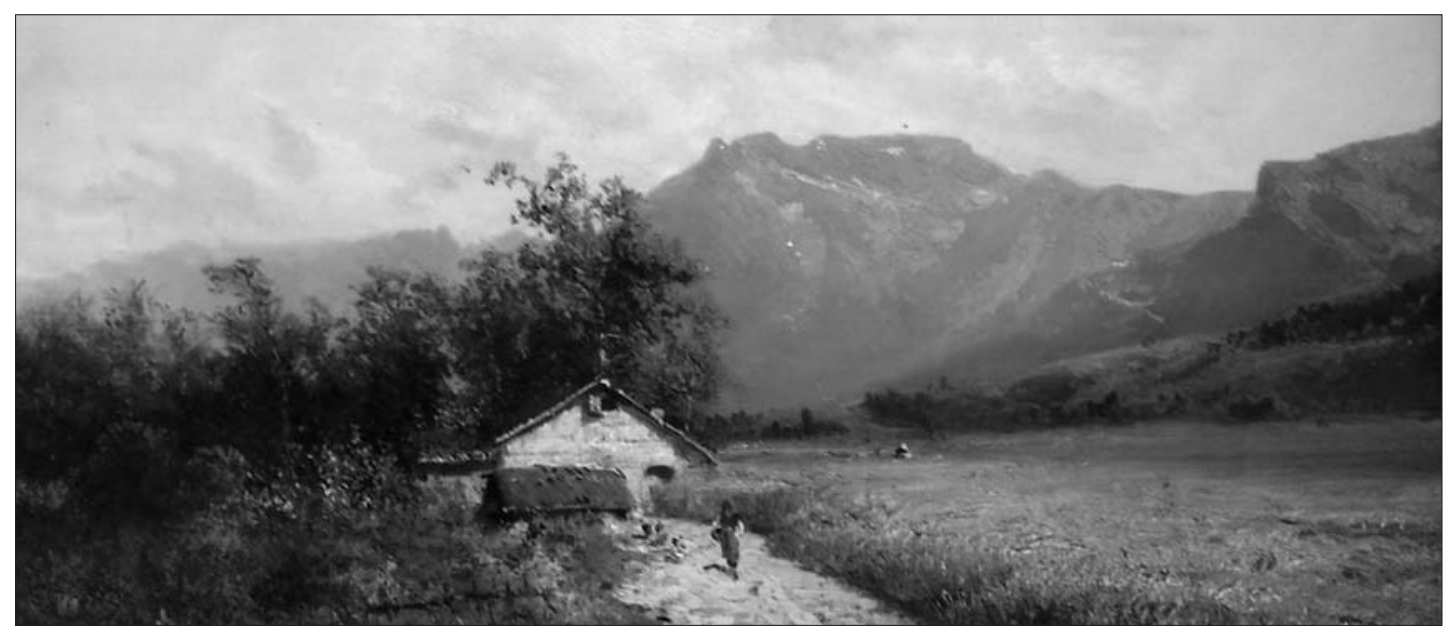

Fig. 11. Carlos Haes: Paisaje montañoso con casa y campo de cereales. Colección particular.

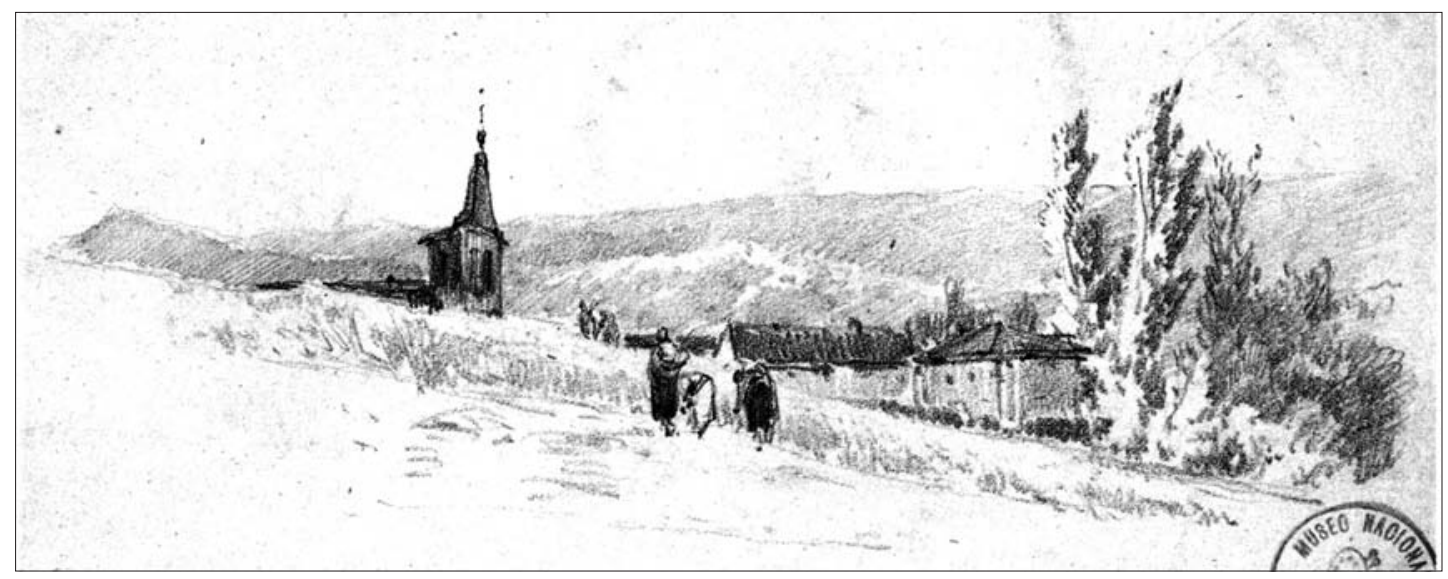

Fig. 12. Carlos Haes: Apuntes en Betelu. Museo del Prado. Madrid. 
Quiñones de León, Vigo, Pontevedra) ${ }^{16}$. No es que sean exactamente iguales y, probablemente, el pico montañoso que aparece en el cuadro que estudiamos no sea Peña Dobres, con que se ha identificado el pico del estudio o boceto, pero es innegable la similitud estructural de ambas disposiciones montañosas. Y no olvidemos que el cuadro que estudiamos es una composición de estudio, lo que puede implicar la algo libre interpretación de los bocetos o estudios utilizados para su realización.

Su fecha de realización, resulta también imprecisa, como en los casos anteriores, por tratarse, muy probablemente, de un cuadro compuesto y ejecutado en el estudio del artista, tomando como base algún boceto o apuntes anteriores. Nos vemos obligados, por tanto, a recurrir nuevamente a la secuencia de tres etapas en que dividieron su obra Beruete y Cid Priego ${ }^{17}$, siguiendo la evolución de su técnica pictórica, según la cual, el jugoso y alegre colorido de este cuadro, junto a lo suelto de su factura, nos hacen pensar que se trate de una obra de su segunda etapa pictórica, fechable, según esto, entre 1870 y 1880.

El otro cuadro, que forma pareja con ese anterior, y que titularemos Paisaje montañoso con casa y campo de cereales (Fig. 11), es igualmente un óleo sobre lienzo de 65 x $92 \mathrm{~cm}$., firmado "C. de Haes" en el ángulo inferior izquierdo. A pesar de las diferencias temáticas de ambos paisajes, la estructura compositiva es básicamente la misma en ambas obras. Un eje central que divide la composición en dos partes. En el cuadro anterior es el torrente, que conduce nuestra vista hacia las montañas del fondo. En este lienzo, el lugar del torrente lo ocupan el camino -por el que discurre una campesina-, la casa en donde ese desemboca y la arboleda que queda detrás de ella; sólo que aquí, el eje que marcan estos elementos no conduce nuestra mirada directamente hacia las montañas del fondo, sino que es algo oblicuo y, en un juego algo barroco, esta diagonal se corta bruscamente en la fachada de la casa y la arboleda de detrás, introduciendo así en la composición un elemento dinámico de distorsión, que nos obliga a desviar nuestra mirada, para buscar las lejanías montañosas, hacia la mitad derecha del cuadro, a través del campo de cereales y el matizado juego del colorido, en luces y penumbras, de las colinas de los términos medios de la composición.

Cuadro de esencia menos bravía que los anteriores, de carácter más apacible y bucólico, suavizado por el variado, rico y cálido colorido que detenta, nos muestra también esa composición armónica, equilibrada y serena -a la que antes aludimos como característica de este tipo de cuadros de Haes-, manteniendo la consonancia de líneas verticales y horizontales en la estructuración de la obra, complementándose con esa serenidad ambiental determinada por el estudio atmosférico y lumínico que establece así las suaves gradaciones de las lejanías, todo ello envuelto en una poética de exquisito lirismo.

Existen también correspondencias de elementos de este cuadro con ciertas representaciones en algunos de los dibujos que conocemos del artista, como son los titulados Un camino con dos figuras (D-5224, Museo del Prado. Madrid) y Apunte en Betelu (Fig. 12) (D-5220, Museo del Prado. Madrid) ${ }^{18}$, cuya concepción y composición guardan relación y parecido con las de este lienzo. Pero es, especialmente, este último dibujo de Apunte en Betelu, sobre el que queremos reclamar especialmente la atención, pues, salvando las lógicas diferencias de elementos compositivos entre las dos obras, no hay duda de que la estructura general de ambas posee ciertas similitudes y, sobre todo, la concepción de los personajes femeninos caminando por el campo con la arquitectura de la iglesia al fondo, coincide con la de la figura de mujer caminando hacia la casa campestre de este lienzo; figura ésta que, además, recuerda enormemente a las del citado dibujo, por su misma concepción y realización; diríase que la figura femenina del óleo está casi copiada de la otra figura femenina de pié del dibujo del Museo del Prado.

ENRIQUe ARIAS-ANGLÉS (CSIC)

\footnotetext{
16 Ibid., pp.92-93 (nº 7391).

17 Vid. Notas 5 y 6.

18 Gutiérrez MÁrquez, A., Carlos de Haes en el Museo del Prado (1826-1898).Catálogo razonado. Museo Nacional del Prado, Madrid, 2002, pp. 128-129 (nº D-5224), y pp. 140-141 (n ${ }^{\circ}$ D-5220).
} 